\title{
Deep brain stimulation for refractory epilepsy
}

Tomasz Tykocki ${ }^{1}$, Tomasz Mandat ${ }^{2}$, Anna Kornakiewicz ${ }^{3}$, Henryk Koziara² ${ }^{2}$ Paweł Nauman ${ }^{1}$

1Department of Neurosurgery, Institute of Psychiatry and Neurology, Warsaw, Poland 2Department of Neurosurgery, Institute of Oncology, Warsaw, Poland

${ }^{3}$ First Faculty of Medicine, Medical University of Warsaw, Poland

Submitted: 18 September 2011

Accepted: 3 December 2011

Arch Med Sci 2012; 8, 5: 805-816

DOI: 10.5114/aoms.2012.31135

Copyright @ 2012 Termedia \& Banach

\author{
Corresponding author: \\ Tomasz Tykocki MD \\ Department of Neurosurgery \\ Institute of Psychiatry \\ and Neurology \\ 9 Sobieskiego St \\ Warsaw 02-957, Poland \\ Phone: +48509764635 \\ E-mail: ttomasz@mp.pl
}

\begin{abstract}
Deep brain stimulation (DBS) is a method of treatment utilized to control medically refractory epilepsy (RE). Patients with medically refractory epilepsy who do not achieve satisfactory control of seizures with pharmacological treatment or surgical resection of the epileptic focus and those who do not qualify for surgery could benefit from DBS. The most frequently used stereotactic targets for DBS are the anterior thalamic nucleus, subthalamic nucleus, central-medial thalamic nucleus, hippocampus, amygdala and cerebellum. The DBS is believed to be an effective method of treatment for various types of epilepsy among adults and adolescents. Side effects may be associated with implantation of electrodes and with the stimulation itself. An increasing number of publications and growing interest in DBS application for RE may result in standardization of the qualification and treatment protocol for RE with DBS.
\end{abstract}

Key words: deep brain stimulation, epilepsy, refractory.

\section{Introduction}

Epilepsy is a common neurological disorder with a prevalence of $0.5-1 \%$ in the general population [1]. More than one third of patients do not achieve a satisfactory clinical effect, despite optimal pharmacotherapy [2]. New antiepileptic drugs (AEDs), which have been introduced to the market in recent years, enable seizure control in another $7 \%$ of patients [3]. Patients diagnosed with refractory epilepsy (RE) may qualify for neurosurgical treatment. However, it has been estimated that over $40 \%$ of patients with RE do not meet the eligibility criteria for the surgical treatment [2]. The risk of serious postoperative complications following neurosurgical resection is estimated to be about $4 \%$. There has been an increasing interest in application of deep brain stimulation (DBS) in the treatment of RE in recent years. The DBS is considered to be a safe and reversible method in treatment of movement disorders, neuropathic pain and psychiatric disorders. Since the 1970s, stereotactic ablation of the basal ganglia has been applied in the treatment of RE in single groups of patients. Despite initially encouraging results, neither recommendation nor inclusion criteria for DBS in RE have been determined. Pioneers of neuromodulation in epilepsy were Adrian Upton, Irvin Cooper and Francisco Velasco, who successfully applied DBS of the anterior nucleus of the thalamus (ANT) in temporal lobe epilepsy and Lennox-Gastaut syndrome (LGS) in the 1980s [4-6]. 
The DBS in the treatment of RE is based on two neuromodulatory mechanisms dependent on stimulated targets: the first one is a gating mechanism, which inhibits the propagation of epileptiform discharges, and the second one is related to direct stimulation of the epileptic focus.

The authors present an analysis of the DBS procedure in the treatment of RE referring to different anatomical targets, the theoretical basis of the antiepileptic mechanism of action, and an evaluation of the efficacy of this method.

\section{The pathomechanism of refractory epilepsy}

The main mechanisms underlying the resistance to medical therapy in epilepsy are: i) impairment of drug penetration into the brain by the bloodbrain barrier [7], and ii) reduced pharmacosensitivity to antiepileptic drugs at the brain receptor level [8]. Overexpression of P-glycoprotein, the product of the multidrug resistance- 1 gene, is observed at the blood-brain barrier in refractory epilepsy, resulting in decreased concentration of the drug in brain tissue, below the therapeutic level [9].

Another important hypothesis is the alternation in the sensitivity of ion channels, in particular the sodium ones, to antiepileptic drugs acting by channel blocking, contributing to lack of activity of these agents [10]. Similar reorganization of $\mathrm{GABA}_{A}$ receptor subunits was found in patients with temporal lobe epilepsy, reducing the antiepileptic potential of GABAergic drugs [11]. However, novel approaches in the treatment of RE emphasize the role of neuroplasticity and remodeling of neural circuits. New genes expressed in the cytoskeleton and synaptic plasticity have been identified in brain tissue of RE patients [12]. The majority of these pathological genes are associated with the growth cone, a very dynamic structure at the tip of the axon, responsible for circuit formation and neuroplasticity, axon outgrowth and regeneration [13]. New neural connections develop by activation of integrins and microtubule-associated proteins and steer the growth cone towards non-physiological direction. The plastic adaptive changes following recurrent seizures consolidate the pathological circuits, resulting in structural and functional abnormalities, such as axonal sprouting, synaptic reorganization, neurogenesis and gliosis, contributing to RE [14]. Specific conditions were identified in the subgranular zone of the dentate gyrus in the hippocampus, where neurogenesis of granule cells was observed after a seizure. Mechanisms underling recruiting newborn neurons to epileptic circuits and propagation of discharges are: formation of hilar basal dendrite and the ectopic migration of progenitor granule cells into the hippocampal hilus [15].

Noteworthy is modulation of astrocytes and gliosis formation after recurrent seizures. There has been found some abnormal expression of proteins such as nestin, aquaporin-1, slit-2, and TGF- $\beta 1$ that appear under pathological injuries such as seizure. Therefore, altered astrocytes disturb the interneural and astrocyte-neuron interaction, promoting the development of RE [16-18].

Other potential mechanisms leading to RE are proinflammatory mediators and neuronal damage. Recurrent seizures induce (IL)-1 $\beta$, IL-6, or TNF, complement, and cyclooxygenase-2, affecting the blood-brain barrier and increasing neuronal hyperexcitability, and neuronal degeneration [19]. Another interesting hypothesis is enhanced hypoxia and $\mathrm{pH}$ shift towards higher acidity in the area of the epileptic focus, further hypoperfusion and lactate production due to recurrent discharges. The implication of this process is the change of gene expression and increased production of hypoxia-inducible factor inducing acidification, and activation of $\mathrm{Na}^{+}$channels, leading to a lowered threshold for neural excitability [20].

\section{The antiepileptic mechanism of action of deep brain stimulation}

There have been distinguished several anatomical targets employed during DBS for RE: the anterior thalamic nucleus, the subthalamic nucleus (STN), the cerebellum (Ce), the hippocampus (Hip), the central-medial nucleus of the thalamus (CMN), the caudate nucleus or the epileptic focus (EF). Depending on the target, the antiepileptic mechanism of action is divided into a gating mechanism of the epileptic discharges and direct inhibition of EF (Figure 1).

The basal ganglia, as part of the cortico-basal ganglia loop, are involved in the modulation and propagation of the epileptic discharges. During electrical or pharmacological stimulation of Hip with the induction of seizures, increased activity of STN, the striatum (St), the thalamus, and the globus pallidus (GP) is observed [21]. Moreover, it has been shown that there is increasing accumulation of 2-deoxyglucose in the basal nuclei during propagation of temporal discharges [22]. Bilateral inhibition of excitatory glutamatergic neurons in the pars reticularis of the substantia nigra (SNr) reduces or eliminates seizures induced by pilocarpine, kainic acid (KA) or flurothyl. This effect occurs only when the rostral part of the SNr is inhibited, whereas activation of GABA neurons of the caudal part of the SNr lowers the seizure threshold [23]. Anticonvulsant activity of $\mathrm{SNr}$ is correlated with the excitation of both GABAergic and serotonergic neurons. The SNr functions as a gating system for spreading of paroxysmal discharges; however, blocking of GABA and 5-HT transmission does not cause epileptic seizures [24]. Only direct administration of KA into the SNr induces seizures, most 


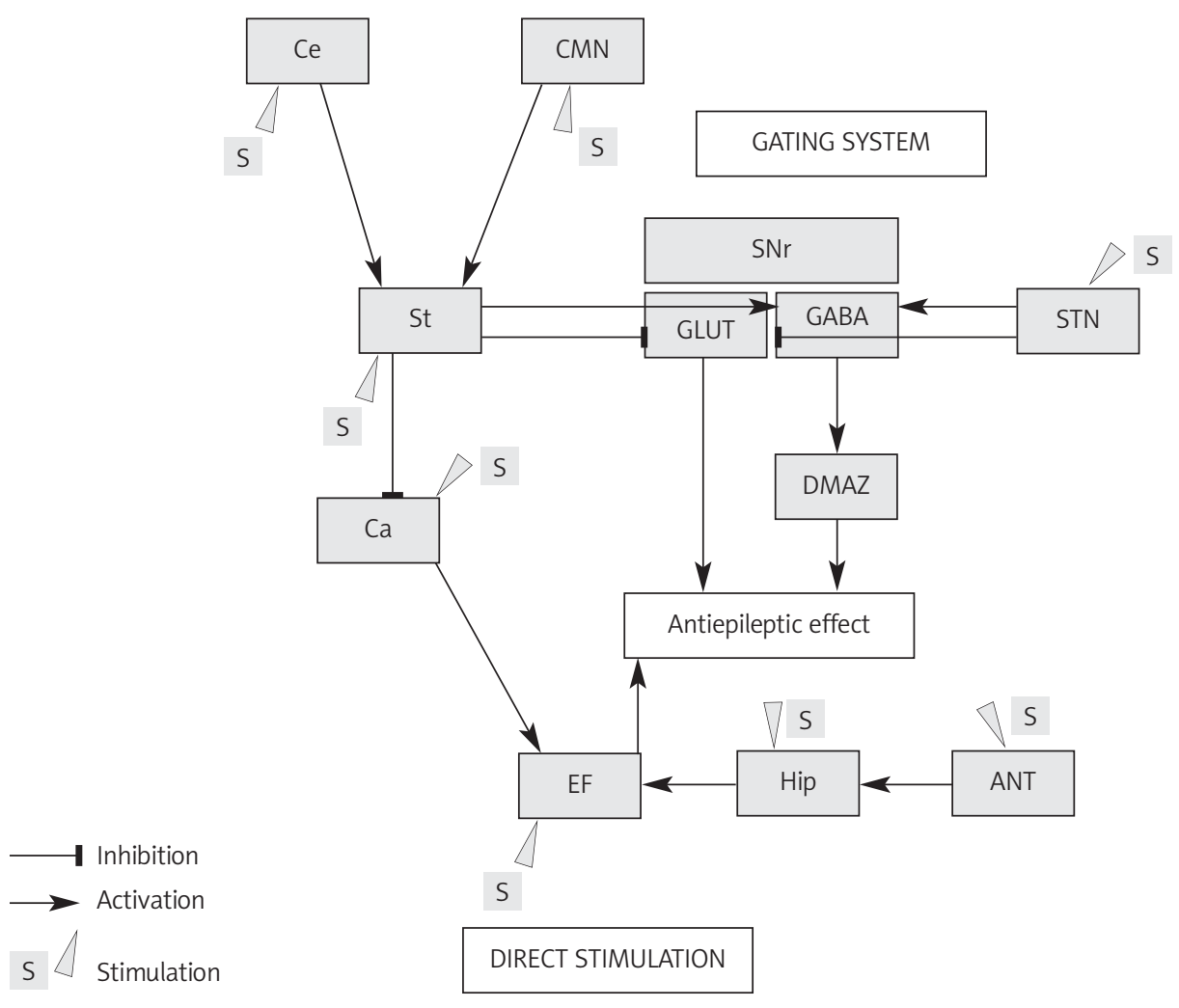

Figure 1. Neuroanatomical basis of antiepileptic mechanism of DBS for different anatomical targets

$\mathrm{Ce}$ - cerebellum, CMN - central-medial nucleus of the thalamus, St - striatum, SNr-pars reticularis of substantia nigra, STN - subthalamic nucleus, CA - corpus amygdaloideum, DMAZ - dorsal midbrain anticonvulsant zone, EF-epileptic focus, Hip - hippocampus, ANT - anterior nucleus of thalamus

likely by the stimulation of the population of neurons which are connected to the limbic system, but the seizures are intractable to the action of GABA agonists [25].

The $\mathrm{SNr}$ receives a direct GABAergic modulation from St, whose activity remains under control of the glutaminergic input from the cerebral cortex and the STN [26]. Electrical or pharmacological stimulation of St immediately interrupts generalized seizures induced by irritation of the amygdalae (corpus amygdaloideum - CA) [27]. In laboratory tests, electrical stimulation or administration of GABA agonists directly into internal globus pallidus (GPi) has not induced equally effective anticonvulsant response as for $\mathrm{SNr}[18,28]$.

The STN plays a role in the modulation of the cortico-basal-thalamocortical connections, which is used in the treatment of movement disorders with DBS. Bilateral STN DBS has been proven to have an effective anti-epileptic mechanism of action which is based on the inhibition of SNr activity by interruption of glutamatergic transmission and selective excitation of GABAergic neurons [29]. Unilateral stimulation of STN inhibits propagation of epileptic discharges in the frontal lobe. This leads to a local antidromic inhibition of afferent fronto-subthalamic connections. However, only bilateral high frequency stimulation (HFS) evokes the inhibition of SNr and suppresses seizures. The data from EEG and LFP (local field potentials) have confirmed the presence of seizure activity of STN corresponding to the side of the frontal epileptic focus [30].

The SNr is connected to the dorsal midbrain anticonvulsant zone (DMAZ) formed by deep and intermediate layers of the superior colliculi (SC), the intercollicular nucleus and the midbrain reticular formation. The DMAZ is inhibited tonically via GABAergic neurons localized in SC and SNr. Therefore blockage of GABA receptors in these structures disinhibits the DMAZ and consequently triggers a strong anticonvulsant effect in case of focal, tonic-clonic (TCS) and absence seizure (AS) [31, 32].

Described in 1937 by Papez [33], neural loops link the hippocampus via the fornix and the mamillary bodies to ANT. In laboratory tests in which seizures were induced by pentylenetetrazol, an effective anticonvulsant response was obtained by HFS of ANT or the mammillothalamic fasciculus. In contrast, during low frequency stimulation (LFS) and after administration of GABA antagonists, the opposite effect was achieved [34].

In pathophysiology of AS a key role is played by the thalamus, which is an important element of the cortico-basal-thalamocortical loop. Neuronal dysfunction of the intralaminar nuclei of the thal- 
amus is responsible for generation of AS with characteristic $3 \mathrm{~Hz}$ spike-wave discharges in EEG. Physiological activity of neurons in AS is similar to that observed during non-rapid eye movement sleep (NREM). The nuclei of the thalamus display tonic activity during REM sleep and wakefulness and cyclic discharge activity during NREM sleep [35]. Propagation of generalized, bilateral and cyclic discharges typical for AS is associated with impaired T-type calcium channels and dysfunction of GABAergic receptors [36].

In 1987, Velasco et al. [6] applied CMN stimulation in patients with RE. This structure belongs to the group of the intralaminar nuclei of the thalamus, projects efferent fibers primarily to $S t$, and receives afferent input from the cerebral cortex, GP, the vestibular nuclei, SC and the reticular formation. The CMN LFS induces absence seizures, while its bilateral damage leads to mutism [37]. Ce stimulation is also used in the treatment of RE, as HFS activates Purkinje cells which have an inhibitory effect on efferent projections to the thalamus $[38,39]$.

Hip and CA stimulation directly inhibits the epileptic focus. Unilateral or bilateral HFS of these structures is effective in the treatment of partial seizures (PS) and secondary generalized seizures (SGS). In laboratory tests it has been found that inhibition of EF activity depends on depolarization block induced by inhibition of voltage-gated calcium channels and hyperpolarization by a significant increase in the concentration of potassium ions in the extracellular space of the hippocampal formation CA1 cells [40].

\section{Patient selection for DBS surgery}

The DBS surgery is performed among patients with the diagnosis of RE; however, the definition of RE has been changing constantly. The International League Against Epilepsy (ILAE) classifies RE as a failure of adequate trials of two tolerated and appropriately chosen AEDs, whether as monotherapy or in combination, to achieve sustained seizurefree response [41]. The seizure-free period in RE is proposed to be less than 12 [2] or 18 [42] months. Currently there is no specific recommendation on DBS treatment for RE issued by neurosurgical and neurological societies.

In the SANTE multi-center, randomized trial [43] ANT DBS has been validated. The patients eligible for SANTE demonstrated partial seizures (PS) or simple partial seizures (SPS) with significant impairment of quality of life due to epilepsy. The occurrence of frequent falls and injuries and the use of at least three AEDs in mono-or polytherapy were consecutive inclusion criteria for SANTE. Among a selected group of patients the seizure frequency (SF) ranged from 6 per month up to 10 per day. The patients treated earlier by vagus nerve stimu- lation or epileptic focus resection were also incorporated in the study. The exclusion criteria for SANTE were: other systemic or neurological disorders, cancer, neurodegenerative disorders, nonepileptic seizures, IQ below 70, inability to undergo neuropsychological assessment and keep seizure diary, or pregnancy. Before the surgery, video-EEG registration and magnetic resonance imaging (MRI) at 1.5 and 3T was performed, and the patients were recommended to observe seizures for 9 months prior to surgery [44].

\section{Deep brain stimulation procedure}

The DBS procedure for RE proceeded in a similar way as in the treatment of movement disorders. On the day of surgery a stereotactic frame was fixed to the head and computed tomography (CT) or MRI scan of the brain was performed. The coordinates for different targets were as follows: ANT: 5-6 mm lateral to the midline $(\mathrm{ML}), 12 \mathrm{~mm}$ above the $A C-P C$; $8 \mathrm{~mm}$ rostral to $\mathrm{PC}[45,46]$; the caudal zona incerta (CZi): $2 \mathrm{~mm}$ below the AC-PC, $6 \mathrm{~mm}$ rostral to the mid-commissural point (MC), $13 \mathrm{~mm}$ lateral to the ML; the posterior hypothalamus (pHyp): $5 \mathrm{~mm}$ below the AC-PC, $6 \mathrm{~mm}$ caudal to the MC, $13 \mathrm{~mm}$ lateral to the $M L$ [47]; STN: $6-7 \mathrm{~mm}$ rostral to the PC, 4-5 mm below the AC-PC, $8 \mathrm{~mm}$ lateral to the $M L$ [48]; $C M N 13 \mathrm{~mm}$ lateral to the $M L$, at the AC-PC level, the anterior edge of the PC [49]. During cerebellar stimulation the electrode was placed $2 \mathrm{~cm}$ lateral to the ML on the superomedial surface of the upper lobe [50].

Intraoperative microrecording or trail macrostimulation was used depending on the neurosurgical center. The AED administration was reduced or withdrawn within 48-72 $\mathrm{h}$ before surgery $[44,51]$. The surgery was performed under general or local anesthesia. Pre-stimulation LFS was carried out to initiate epileptic discharges and the effect was recorded by EEG and ECOG.

Boon et al. [44], during functional amygdalohippocampectomy, have implanted electrodes unilaterally into Hip and CA. During the procedure they stimulated one of the targets, and recorded local field potentials (LFP) from another one at the same time. Threshold stimulation was determined when artifacts in LFP appeared. Initiation of stimulation was performed one month after implantation.

\section{Side effects and risks of deep brain stimulation}

Side effects associated with DBS may be due to the neurosurgical procedure itself, e.g. implementation of electrodes and pacemaker, or occur secondarily to stimulation. In the SANTE study, the mortality rate was $2.7 \%(3 / 110)$ in a 3-year follow-up. No deaths were associated with surgery and did not occur in the post-operative period 
(up to 3 months after implantation). In 2 cases, SUDEP (sudden unexplained death in epilepsy) occurred. $12.7 \%$ of patients experienced infectious complications, and $4.5 \%$ of the cohort suffered from symptomatic intracerebral hemorrhage. Improper electrode insertion outside ANT, visible in the postoperative MRI, were found in $8.2 \%$ of patients. In 5 patients status epilepticus was reported regardless of stimulation. The complication rate was similar to that observed in movement disorders treated with DBS. Complications after DBS surgery for Parkinson's disease associated with pacemaker damage, migration or fracture of intracerebral electrodes and erosion of skin over the pacemaker affects 1 in 4 patients operated on (for a mean observation time of 17 months) [52]. The side effects associated with stimulation include paraesthesia, depression, seizures different from preoperative ones and memory disorders. Previous studies, prior to SANTE, have drawn attention to the risk of memory disorders and mood swings after stimulation of the hippocampus and the amygdala [53]. More recent studies have shown that the functional amygdalohippocampectomy for RE with constant HFS (130$200 \mathrm{~Hz}$ ) does not impair cognitive function and does not cause emotional disorders [54].

The overall summarized complication rate concerning deep brain stimulation is presented below. Incidence rate of symptomatic subdural hemorrhages is less than 1\% [55]. Symptomatic intracerebral hemorrhage has been reported to occur in $1-5 \%$ of cases [56]. Intraoperative or post-operative seizures, due to localized ischemia and/or hemorrhagic cortical irritation, is estimated to be comparable to the risk of seizure after ventricular shunt placement, which has a rate of less than $5 \%$ in the first year. A recent review reports incidence rates up to $14 \%$ and overall risk approximately $2.4 \%$ [57]. The incidence of venous infarction is $0.9 \%$ [58]. Pulmonary embolism occurring in the early postoperative setting after neurosurgical procedures ranges from 0.4 to 4.9 [59]. There is a $4-12.2 \%$ risk per patient or $1.5-9.7 \%$ risk per lead of perioperative infection [60]. Superficial infections appear to be the most common. The combined deep infection and erosion rates have been reported at 1.5-15.2\% [61] and the rate of lead fracture at 2.0-9.9\% per patient, or $\sim 1.8 \%$ per lead implanted [62]. Lead fracture may cause a short or open circuit, which has been reported to occur in $0.9-9.9 \%$ of patients [63]. Overall hardware complications occur in $7.8 \%$ to $32.9 \%$ of patients from misplaced lead, loss of effect, lack of efficacy, lead fracture, lead migration or infection [64].

\section{Results of deep brain stimulation for epilepsy}

The reduction of seizure frequency after ANT DBS may range between 46 and $67 \%$ [43, 45, 46, 65-67]
(Table I). The HFS is applied at a minimum frequency of $90 \mathrm{~Hz}$ with a cyclic stimulation (1 min ON, 5 min OFF) and pulse width of 60-90 $\mu \mathrm{s}$. In the randomized double blind SANTE study the effect of bilateral ANT DBS for RE has been evaluated. A month after implantation of electrodes, some patients were included in the group subjected to stimulation (stimulated group - SG) $(n=54)$, and other patients were assigned to the control group (control group - CG) $(n=55)$. The frequency of SF was assessed in a 3-month period, and after that all patients were stimulated. A month after surgery, before randomization, when the stimulation was switched off in all patients, SF decreased on average by $20 \%$. The improvement could be explained by the microlesion effect appearing after surgery. In the first month after randomization, in 1 patient from SG, 210 seizures was reported, which resolved when the stimulation had been stopped. Re-stimulation with the voltage reduction from $5 \mathrm{~V}$ to $4 \mathrm{~V}$ did not cause seizure recurrence. Reduction of SF in the first, second and third month of the double-blind trial was as follows: SG $33.9 \%$, $42.1 \%, 40.4 \%$ and $25.3 \%$ CG, $28.7 \%, 14.5 \%$.

Improvement in patients with partial complex seizure (PCS) was $36.3 \%$ for SG and $12.1 \%$ for CG and the percentage of seizure-related injuries was $26 \%$ and $7 \%$ respectively. Among patients with temporal lobe epilepsy a better result was obtained in SG in comparison to CG, whereas for the patients with frontal, parietal or occipital epilepsy there were no statistically significant differences between the groups. After initiation of stimulation in all subjected patients, SF reduction reached $41 \%$ at 13 months and $56 \%$ at 25 months. In 16\% (13/81) of patients a decrease in SF by more than $90 \%$ was reported, 8 patients were seizure free for a year, and 4 for 2 years. However, in 3 patients, SF increased by more than $50 \%$ after DBS.

Baseline parameters of the stimulation were $5 \mathrm{~V}, 145 \mathrm{~Hz}, 90 \mu \mathrm{s}$, cyclic stimulation (1 min ON, 5 OFF), and it was modified depending on the observed side effects. An increase in voltage up to $7.5 \mathrm{~V}$ and frequencies up to $180 \mathrm{~Hz}$ did not improve seizure control. Comparison of the clinical results after STN DBS and ANT DBS for RE, made by the same team, has shown ANT DBS to be more effective than STN DBS. A decrease in SF by $75 \%$ and $49 \%$ was found respectively [68]. Implantation of electrodes itself, without any stimulation, caused $20 \%$ to $90 \%$ seizure reduction in patients with RE [43, $45,68]$. That may result from the microlesion effect. In the SANTE study SF reduction was observed up to 4 months after surgery in both groups when stimulation was off and after this period the number of seizures increased in CG, whereas in SG a further seizure reduction was observed when the stimulation was on. Hodaie et al. [45] have reported 
Table I. Comparison of the results of deep brain stimulation for different anatomical targets

\begin{tabular}{|c|c|c|c|c|c|}
\hline Target & Authors & Year & $\begin{array}{l}\text { Number } \\
\text { of patients }\end{array}$ & $\begin{array}{l}\text { Additional features/ } \\
\text { type of seizures }\end{array}$ & $\begin{array}{l}\text { Results - reduction } \\
\text { of seizure frequency }\end{array}$ \\
\hline \multirow[t]{7}{*}{ ANT } & Hodaie et al. & 2002 & 5 & PCS, TCS, AS, AT & $54 \%$ \\
\hline & Kerrigan et al. & 2004 & 5 & PSC, TSC & $14.2 \%$ \\
\hline & Osorio et al. & 2007 & 4 & MTL & $75.6 \%$ \\
\hline & Lim et al. & 2007 & 4 & PSC, SG & $49 \%$ \\
\hline & Andrade et al. & 2006 & 6 & TCS, SG & $\geq 50 \%$ \\
\hline & SANTE study & 2010 & 110 & SGS, PCS & $56 \%$ \\
\hline & Beox & 2011 & 8 & MTL epilepsy, HS & $65-75 \%$ \\
\hline $\begin{array}{l}\text { ANT } \\
\text { vs. STN }\end{array}$ & Lee et al. & 2002 & 6 & SGS, PCS & ANT $-75 \%$, STN $-49 \%$ \\
\hline \multirow[t]{9}{*}{ STN } & Benabid et al. & 2002 & 1 & Cortex dysplasia in parietal lobe & $80 \%$ \\
\hline & \multirow[t]{3}{*}{ Chabardès et al. } & \multirow[t]{3}{*}{2002} & 3 & PCS & $67-80 \%$ \\
\hline & & & 5 & $\begin{array}{c}\text { Autosomal dominant frontal lobe epilep } \\
\text { with insulo-frontal seizures }\end{array}$ & psy \\
\hline & & & 1 & Dravet syndrome & $40 \%$ \\
\hline & Vesper et al. & 2007 & 1 & Myoclonic seizures & $50 \%$ \\
\hline & Shon et al. & 2005 & 2 & TS & $90 \%$ \\
\hline & \multirow[t]{2}{*}{ Handforth et al. } & \multirow[t]{2}{*}{2006} & 1 & Left frontal encephalomalacia & $55 \%$ \\
\hline & & & 1 & PCS & $33 \%$ \\
\hline & Wille & 2011 & 5 & Myoclonic seizures & $\approx 50 \%$ \\
\hline \multirow[t]{5}{*}{ MTL } & Boon et al. & 2007 & 12 & MTL epilepsy & $\approx 50 \%$ \\
\hline & Tellez-Zenteno et al. & 2006 & 4 & MTL epilepsy, SPS, PCS, SGS & $15 \%$ \\
\hline & Velasco et al. & 2007 & 9 & Hippocampal sclerosis, PCS, SGS & $\begin{array}{c}5 \text { patients }-95 \% \\
4 \text { patients }-50-70 \%\end{array}$ \\
\hline & McLachlan et al. & 2010 & 2 & MTL epilepsy & $33 \%$ \\
\hline & Miatton et al. & 2011 & 10 & MTL & $53-57 \%$ \\
\hline \multirow[t]{5}{*}{ CMN } & Velasco et al. & 1987 & 15 & $\begin{array}{l}\text { SGS, LGS } \\
\text { PCS }\end{array}$ & $\begin{array}{l}80 \% \\
<50 \%\end{array}$ \\
\hline & Fishera et al. & 1992 & 7 & TCS & $30 \%$ \\
\hline & Velasco et al. & 2000 & 13 & & $>50 \%$ \\
\hline & Cukiert et al. & 2009 & 4 & SGS & $65-95 \%$ \\
\hline & Valentin et al. & 2011 & 1 & Status epilepticus & $100 \%$ \\
\hline \multirow[t]{4}{*}{ CeS } & Cooper et al. & 1973 & 15 & SGS & $\geq 50 \%$ \\
\hline & Davis et al. & 1992 & 27 & Spastic seizures & $25-67 \%$ \\
\hline & Wright et al. & 1984 & 12 & $\begin{array}{l}\text { Grand mal, AS, PCS, } \\
\text { Myoclonic jerks }\end{array}$ & $\begin{array}{l}\text { No statistically } \\
\text { significant reduction. }\end{array}$ \\
\hline & Velasco et al. & 2005 & 5 & TCS, TS & $61-76 \%$ \\
\hline pHyp & \multirow[t]{3}{*}{ Franzini et al. } & \multirow[t]{3}{*}{2008} & 2 & Multifocal epilepsy & $75 \%, 80 \%$ \\
\hline \multirow[t]{2}{*}{ Czi } & & & 2 & SPS, SGS & $85 \%$ \\
\hline & & & & Rasmussen's syndrome & $\begin{array}{l}\text { Disappearance } \\
\text { of the status } \\
\text { epilepticus at } \\
\text { 6-month observation }\end{array}$ \\
\hline NC & Chkhenkeli et al. & 1997 & 57 & PCS, SGS & $\begin{array}{l}\text { Reduction at } 4-8 \mathrm{~Hz} \\
\text { increase at } \mathrm{HFS}\end{array}$ \\
\hline
\end{tabular}


reduction in SF after implantation of electrodes without stimulation $(n=5)$, but DBS had no effect on further reduction of SF. There was no difference for SF between using constant and cyclic stimulation, and no DBS impact on IQ scores in psychological tests [68]. ANT DBS was proved to be effective in hippocampal sclerosis; a significant decrease $(65-75 \%)$ in seizure frequency was observed in 2 patients [69].

Unilateral STN DBS in a 5-year-old patient with cortex dysplasia in the parietal lobe, with a history of 210 seizures per month, resulted in $80 \%$ voltage-dependent seizure reduction (including status epilepticus) in a 30-month follow-up [56]. In another study [70] unilateral and bilateral electrodes were implanted in STN in 9 people. In 3 patients with PCS, including 1 patient previously treated with a prefrontal cortex resection, an SF reduction by 67-80\% was obtained. In a patient with Dravet syndrome DBS caused $40 \%$ improvement in SF, and doses of valproic acid could be reduced due to its serum concentration increase after initiation of stimulation. In all cases a constant stimulation was used with the following parameters: $130 \mathrm{~Hz}$, 60-90 $\mu \mathrm{s}, 1.5-5.2 \mathrm{~V}$. The efficacy of bilateral STN DBS for the treatment of myoclonic seizures in adults has been confirmed [71]. In 2 patients selected for STN DBS due to drug-resistant TS almost $90 \%$ reduction in SF at 18-month follow-up was obtained. These patients were previously treated with resectional therapy. Comparison of the SPECT study performed before DBS and 6 months after the surgery showed an increase in regional blood flow in the dorsolateral and inferior part of the right frontal lobe, in the right superior and inferior temporal gyri and in the epileptic focus [72]. In another study $(n=2)$ improvement following bilateral STN DBS reached $33 \%$ and $50 \%$ [71]. Wille et al. implanted electrodes in the substantia nigra pars reticulata and STN in 5 patients suffering from progressive myoclonic epilepsy. The improvement rate was $30-100 \%$ and was inversely proportional to the severity of the disease [73].

Unilateral or bilateral stimulation of Hip or CA was performed depending on the location of the epileptic focus. The range of stimulation parameters was $130-200 \mathrm{~Hz}, 90-450 \mu \mathrm{s}$, with constant or periodic stimulation ( 1 min ON and 4 min OFF) $[44,49,74,75]$. Boon et al. [76] implanted two electrodes bilaterally in the MTL (mesial temporal lobe). The first was positioned in the CA and the other one in the frontal part of the Hip. Cortical electrodes was implanted for EEG recordings within 48 postoperative hours with no change in the dosage of AEDs and then the recordings were repeated after the suppression of drugs to induce and localize the epileptic focus. Then with the use of an external pulse generator, CA and Hip electrodes were stimulated on the side corresponding to the unilateral epileptic focus. In cases with bilateral epileptic foci, only Hip electrodes on both sides were stimulated. This technique allowed for a $50 \%$ reduction of spike activity in the focal area in EEG within 3 weeks. When there was no effect, the frequency of stimulation was increased to $200 \mathrm{~Hz}$ and observation was continued for another 21 days. In conclusion, at 31-month follow-up, MTL DBS was performed in 10 of 12 patients and 2 of them underwent surgical resection. Parameters of stimulation

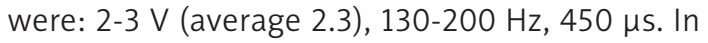
5 patients SF was reduced by half, in two cases by less than $50 \%$, in 1 patient no improvement was noted and in another patient no seizure was observed during follow-up. A double-blind, multiple cross-over, randomized study, designed to analyze the ON and OFF Hip stimulation effect $(n=4)$ for SF, showed an ON/OFF difference of $15 \%$. In the neuropsychological tests there was no difference between patients with DBS ON and OFF [75]. The same group reported 2 additional patients randomly assigned to 3 months on and off stimulation trials. The reduction of SF was 33\% [77]. In the results of Miatton et al. $(n=10)$ improvement after amygdalohippocampal DBS was over 50\% [78].

Velasco et al. applied Hip DBS in patients with hippocampal sclerosis (HS) diagnosed with MRI $(n=4)$. Among patients with HS reduction in SF was noted not earlier than 8 months after initiation of stimulation and this reduction was lower than in patients without HS, in whom effects had been observed in the first month after the stimulation was switched on. In this study, 1 month after implantation all patients were divided into groups: the first one with DBS ON $(n=4)$ and the second one with DBS OFF ( $n=5$, including patients with HS: $n=2$ ). In the OFF group there were no differences in SF in comparison to the pre-operative condition; however, in 1 patient with HS, the seizure frequency doubled. Decreased and delayed response to DBS in patients with HS may result from increased electrical resistance of the tissue as well as reduced benzodiazepine receptor density [49].

Another anatomical target, the CMN, is part of the intralaminar nuclei of the thalamus responsible for cortical desynchronization [79]. During bilateral CMN DBS in 15 patients [80] with PCS, SGS, TS and LGS stimulation parameters were $60 \mathrm{~Hz}$, 4-6 V, 400-600 $\mu \mathrm{s}$, cyclic stimulation: $1 \mathrm{~min}$ ON and 4 min OFF between the left and right side. After 6 months of stimulation patients were divided into groups ON and OFF, and effects of stimulation were evaluated using a double-blind trial. In patients with LGS or SGS $80 \%$ reduction in SF following stimulation was obtained. A lower effect $(<50 \%)$ was observed in the group with PCS, particularly with temporal lobe epilepsy. In another study, reduction 
of SF by CMN DBS in the OFF group was lower than before the operation [81]. The improvement after re-stimulation was earlier, when the ON period before turning off was longer. The antiepileptic effect may be related to time-dependent modulation of neuronal stimulation by DBS. A better response was obtained during stimulation of the ventro-lateral part of the CMN than the dorso-medial posterior. In the study of Fisher et al. [82] CMN DBS in 7 patients with TCS decreased SF by $30 \%$ in ON and $8 \%$ in OFF stimulation conditions. The best clinical responders were patients with TCS, LGS, SGS. In another study SF reduction in 4 patients with SGS ranged from $65 \%$ to $95 \%$. Interictal spiking frequency was reduced from $25 \%$ to $95 \%$, but their morphology remained the same [83]. Promising improvement was observed in a case of vegetative state with generalized status epilepticus; all the epileptic activity disappeared after 4 weeks [84].

In the 1970s, Cooper et al. [71] applied cerebellar stimulation (CeS) in patients with RE. In 66\% of patients (10/15) SF decreased at least by half over the 3-year follow-up. Stimulation of the anterior lobe of the cerebellum in comparison to the posterior lobe proved to be more effective. In histopathological assessment of the cerebellar samples collected during the implantation procedure, fewer Purkinje and cerebellar stellate-like cells were found in comparison to the physiological condition. Davis et al. [70] reported SF reduction in 23 out of 27 patients after CeS (mean follow-up of 14.3 years). Among 12 patients in whom a pulse generator malfunction was found during observation, 5 patients had no seizures, and 7 achieved a significant reduction in seizures. In a randomized, double-blind study ( $n=12$ ) [85] with CeS, consisting of three phases, each lasting 2 months, patients were subjected to constant, on-demand stimulation or no stimulation. There was no statistically significant reduction in SE after CeS. However, according to the patients, the CeS changed their quality of life substantially, seizures became less burdensome, and therefore they expected the treatment to be continued. The results confirmed the evident role of the placebo effect. Although in any phase the patients did not know whether the stimulation was turned on or off, they always reported improvement.

In 2005 the results of a Mexican, randomized study, in which electrodes were implanted bilaterally into the superomedialis hemisphere of the cerebellum of 5 patients, were published [86]. Three months after implantation patients were divided into groups with stimulation turned on and off. There was no statistically significant difference from baseline in the OFF group. In the stimulated group SF decreased to 33\%. After 6 months of CeS the average SF decrease was 61\% (14-75\%) and after 24 months it was 76\% (11-38\%). Improvement in TCS after CeS occurred earlier than in temporal lobe epilepsy. Three patients were re-operated on due to electrode migration.

Favorable effects were observed after bilateral stimulation of the pHyp $(n=2)$, or CZi $(n=2)$ unilaterally [47]. Two patients from the pHyp group with atonic-myoclonic and gelastic seizures with impaired consciousness obtained SF reduction of $75 \%$ and $80 \%$ respectively, whereas implantation of electrodes into the CZi decreased SF by $85 \%$ in a patient with post-traumatic motor seizures at the 4-year follow-up. In a patient with Rasmussen's syndrome, treated with CZi DBS due to status epilepticus, DBS allowed for disappearance of the status epilepticus at 6-month observation. For both targets permanent monopolar stimulation was

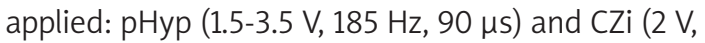
100-185 Hz, $90 \mu \mathrm{s})$. Effectiveness of pHyp DBS may be associated with activation of histaminergic connections with the cerebral cortex [87]. The CZi, having afferent cerebellar projections to GPI and efferent from the basal ganglia, the thalamic nuclei, the midbrain and ascending and activating part of the reticular formation, plays an important role in the control of epileptic discharges [88-90].

In 1997 Chkhenkeli et al. [87] published results on implantation of electrodes into a head of caudate nucleus for epilepsy. The HFS triggered exacerbation of the epileptic discharges, while the 4-8 Hz stimulation resulted in their reduction.

Introduction of the closed loop (closed-loop system, CLS) in the neuromodulatory treatment of RE gave some hope for better control of seizures. The idea of CLS is based on implantation of devices recording EEG and initiating stimulation only after the identification of the epileptic discharges. The responsive neurostimulator system (RNS) [91] consists of a neurostimulator with built-in microprocessor, which is connected to two electrodes placed in deep structures or in the epileptic focus. The stimulator analyzing EEG, which is registered with electrodes, is positioned in the bone of the calvaria and programmed percutaneously. The RNS is programmed to recognize the epileptic discharges based on averaged analysis of frequency and amplitude of the recording in time unit. The range of the stimulation pattern is 1-333 Hz, 1-12 mA, 40-1000 $\mu \mathrm{s}$. Stimulation is performed in volleys lasting up to $5 \mathrm{~s}$, and if seizure is not interrupted, stimulation is repeated. The data on stimulation and EEG are stored in the RNS and on the basis of their analysis the algorithm of stimulation can be adjusted. Data from a randomized, multicentre study ( $n=240)$ have not been published yet, but preliminary reports confirm the efficacy of this method.

The DBS evokes a specific neurophysiological response observed in electroencephalography and may vary according to the frequency of stimulation. 
Cukiert et al. [92] found bilateral cortical recruiting responses (RR) after unilateral ANT $(n=6)$ and CMN $(n=6)$ at low frequency stimulation $(6 \mathrm{~Hz}, 300 \mathrm{~ms})$. RR was diffuse with dominance over the frontotemporal region bilaterally, and over the ipsilateral hemisphere. High frequency stimulation $(130 \mathrm{~Hz}, 300 \mathrm{~ms})$ resulted in background slowing and direct current shift. The mean voltage for the appearance of RR was $4 \mathrm{~V}(\mathrm{CMN})$ and $3 \mathrm{~V}$ (ANT) and neither target influenced inter-ictal spiking frequency or morphology. In the same study 6 other patients were recruited to Hip DBS. The LFS produced RR only in the ipsilateral temporal lobe and HFS abolished inter-ictal spiking. Boëx et al. [93] also reported the advantages of HFS over LFS in RE in short-term observation. Tyrand et al. [94] investigated the effect of two stimulation parameters of the amygdala-hippocampal complex; pseudo monophasic (high amplitude cathodic pulses and very low amplitude anodic pulses) and biphasic (cathodic-negative and anodic-positive pulses) both at $130 \mathrm{~Hz}$, in two groups of epileptic patients: with hippocampal sclerosis (HS) and non-hippocampal sclerosis $(\mathrm{nHS})$. The reduction of interictal epileptic discharge rates (IEDRs) was significant at biphasic stimulation (51.8\%) in comparison to pseudo monophasic (1.57\%) in the HS group, whereas in the $\mathrm{nHS}$ group there was no significant reduction at any stimulation mode.

\section{Ethical aspects of deep brain stimulation}

Deep brain stimulation is a relatively new procedure used in neurosurgery that started in the first half of the 1990s [95]. The DBS is regarded as highly effective in the treatment of Parkinson's disease, dystonia and essential tremor. For each medical treatment, particularly in an invasive approach such as DBS, the key is to counterbalance the potential complications and benefits for the patient. The basis of DBS is to provide electrical current with proper frequency and amplitude to a precisely planned anatomical target, and the procedural technique itself may raise ethical controversy, having regard to the particular nature of brain tissue and its delicacy. In addition, the exact mechanism of action of DBS is still not elucidated and it remains unknown how it affects and modulates complex neural pathways. However, it must be emphasized that DBS is a reversible method and can be terminated at any time. Another important issue is the influence on crucial personality traits of patients such as cognition, emotion and behavior change and despite the improvement of physical symptoms, the final result may lead to deterioration of both social functioning and relationship with the family. They can result from unrealistic expectations of patients and a period of adaptation is necessary for both patients and their families after surgery [96]. Patients undergoing DBS must give their voluntary and fully informed consent for this procedure. However, some patients may insist on undergoing the DBS procedure because of their dramatic situation, faced with a serious, progressive, and incurable disease [97]. An exceptional group is that of children and adolescents, because of sparse data in the literature on the effects of DBS in these patients. Therefore, neuromodulatory treatment should be performed only under specific restrictions and only in experienced centers [98].

Application of DBS in the treatment of RE is still an experimental therapy. There have been reported less than 200 cases with RE and implanted electrodes for different purposes, with a moderate clinical response. Only the SANTE study was randomized and the results may be encouraging in the treatment of RE. However, the study was sponsored by Medtronic, which may raise ethical concerns.

\section{Summary}

The DBS is a safe method with effectiveness proven in the treatment of certain types of RE. Promising results have been obtained in children in whom seizures were associated with CNS malformations. The effect of stimulation may be observed in the first month of treatment, and in the case of HS even several months after implantation. The effectiveness of DBS measured by the reduction of RE remains at a similar level during extended follow-ups. The results has shown a large range of clinical response to DBS, from complete suppression of seizures to their exacerbation. The effectiveness of DBS for different anatomical targets in the treatment of RE confirms a complex mechanism of the propagation of epileptic discharges. Neuromodulation of the basal nuclei favorably affects not only the extrapyramidal symptoms, but also improves the quality of life of patients with epilepsy.

So far no selection algorithm for DBS treatment in patients with RE has been developed and the mechanism of DBS antiepileptic action has not been explained. However, an increasing number of publications and the first multi-center, randomized trial have been giving hope for development of selection and therapeutic algorithms in the treatment of refractory epilepsy with deep brain stimulation.

\section{References}

1. Hauser WA, Annegers JF, Kurland LT. Incidence of epilepsy and unprovoked seizures in Rochester, Minnesota: 19351984. Epilepsia 1993; 34: 453-68.

2. Kwan P, Brodie MJ. Early identification of refractory epilepsy. N Engl J Med 2000; 342: 314-9.

3. Fisher RS. Emerging antiepileptic drugs. Neurology 1993; 43 (11 Suppl): S12-20. 
4. Upton AR, Cooper IS, Springman M, et al. Suppression of seizures and psychosis of limbic system origin by chronic stimulation of anterior nucleus of the thalamus. Int J Neurol 1985-1986; 19-20: 223-30.

5. Velasco M, Velasco F, Velasco AL, et al. Acute and chronic electrical stimulation of the centromedian thalamic nucleus: modulation of reticulo-cortical systems and predictor factors for generalized seizure control. Arch Med Res 2000; 31: 304-15.

6. Velasco F, Velasco M, Ogarrio C, et al. Electrical stimulation of the centromedian thalamic nucleus in the treatment of convulsive seizures: a preliminary report. Epilepsia 1987; 28: 421-30.

7. Kwan P, Brodie MJ. Potential role of drug transporters in the pathogenesis of medically intractable epilepsy. Epilepsia 2005; 46: 224-35.

8. Loscher W, Schmidt D. New horizons in the development of antiepileptic drugs: the search for new targets. Epilepsy Res 2004; 60: 77-159.

9. Tishler D, Weinberg K, Hinton D, Barbaro N, Geralyn A, Corey R. MDR1 gene expression in brain of patient with medically intractable epilepsy. Epilepsia 1995; 36: 1-6.

10. Remy S, Gabriel S, Urban BW, et al. A novel mechanism underlying drug resistance in chronic epilepsy. Ann Neurol 2003; 53: 469-79.

11. Brooks-Kayal AR, Shumate MD, Jin $H$, et al. Selective changes in single cell $\operatorname{GABA}(\mathrm{A})$ a receptor subunit expression and function in temporal lobe epilepsy. Nat Med 1998; 4: 1166-72.

12. Xi ZQ, Xiao F, Yuan J, et al. Gene expression analysis on anterior temporal neocortex of patients with intractable epilepsy. Synapse 2009; 63: 1017-28.

13. Munno DW, Syed NI. Synaptogenesis in the CNS: an odyssey from wiring together to firing together. J Physiol 2003; 552: 1-11

14. Lledo PM, Alonso M, Grubb MS. Adult neurogenesis and functional plasticity in neuronal circuits. Nat Rev Neurosci 2006; 7: 179-93.

15. Parent JM, Elliott RC, Pleasure SJ, et al. Aberrant seizureinduced neurogenesis in experimental temporal lobe epilepsy. Ann Neurol 2006; 59: 81-91.

16. Wang L, Wang XF, Yuan J, et al. Nestin in the temporal neocortex of the intractable epilepsy patients. Neurochem Res 2009; 34: 574-80.

17. Zhou S, Sun X, Liu L, et al. Increased expression of aquaporin-1 in the anterior temporal neocortex of patients with intractable epilepsy. Neurol Res 2008; 30: 400-5.

18. Lu Y, Xue T, Yuan J, et al. Increased expression of TGFbeta type I receptor in brain tissues of patients with temporal lobe epilepsy. Clin Sci (Lond) 2009; 117: 17-22.

19. Vezzani A, Granata T. Brain inflammation in epilepsy: experimental and clinical evidence. Epilepsia 2005; 46: 1724-43.

20. Schaller B. Influences of brain tumor-associated $\mathrm{pH}$ changes and hypoxia on epileptogenesis. Acta Neurol Scand 2005; 111: 75-83.

21. Heath RG. Brain function in epilepsy: midbrain, medullary, and cerebellar interaction with the rostral forebrain. J Neurol Neurosurg Psychiatry 1976; 39: 1037-51.

22. Engel J Jr, Wolfson L, Brown L. Anatomical correlates of electrical and behavioral events related to amygdaloid kindling. Ann Neurol 1978; 3: 538-44.

23. Velísková J, Moshé SL. Update on the role of substantia nigra pars reticulata in the regulation of seizures. Epilepsy Curr 2006; 6: 83-7.

24. Gale K. Evidence for GABA receptors on serotonergic afferent terminals in the substantia nigra and on nigral efferent projections to the caudal mesencephalon. Brain Res 1982; 231: 209-15.

25. Maggio R, Liminga U, Gale K, et al. Selective stimulation of kainate but not quisqualate or NMDA receptors in substantia nigra evokes limbic motor seizures. Brain Res 1990; 528: 223-30.

26. Maurice N, Deniau JM, Glowinski J, et al. Relationships between the prefrontal cortex and the basal ganglia in the rat: physiology of the corticosubthalamic circuits. J Neurosci 1998; 18: 9539-46.

27. La Grutta V, Amato G, Zagami MT. The importance of the caudate nucleus in the control of convulsive activity in the amygdaloid complex and the temporal cortex of the cat. Electroencephalogr Clin Neurophysiol 1971; 31: 57-69.

28. Millan MH, Meldrum BS, Boersma CA, et al. Excitant amino acids and audiogenic seizures in the genetically epilepsy-prone rat. II. Efferent seizure propagating pathway. Exp Neurol 1988; 99: 687-98.

29. Maurice N, Thierry AM, Glowinski J, et al. Spontaneous and evoked activity of substantia nigra pars reticulata neurons during high-frequency stimulation of the subthalamic nucleus. J Neurosci 2003; 23: 9929-36.

30. Usui N, Maesawa S, Kajita Y, et al. Suppression of secondary generalization of limbic seizures by stimulation of subthalamic nucleus in rats. J Neurosurg 2005; 102: 1122-9.

31. Redgrave P, Simkins M, Overton P, et al. Anticonvulsant role of nigrotectal projection in the maximal electroshock model of epilepsy. Mapping of dorsal midbrain with bicuculline. Neuroscience 1992; 46: 379-90.

32. Redgrave P, Marrow LP, Dean P. Anticonvulsant role of nigrotectal projection in the maximal electroshock model of epilepsy. II. Pathways from substantia nigra pars lateralis and adjacent peripeduncular area to the dorsal midbrain. Neuroscience 1992; 46: 391-406.

33. Papez JW. A proposed mechanism of emotion. 1937. J Neuropsychiatry Clin Neurosci 1995; 7: 103-12.

34. Mirski MA, Ferrendelli JA. Interruption of the mammillothalamic tract prevents seizures in guinea pigs. Science 1984; 226: 72-4

35. Steriade M. Corticothalamic resonance, states of vigilance and mentation. Neuroscience 2000; 101: 243-76.

36. Gomora JC, Daud AN, Weiergräber M, Perez-Reyes E. Block of cloned human T-type calcium channels by succinimide antiepileptic drugs. Mol Pharmacol 2001; 60: 1121-32.

37. Watson RT, Valenstein E, Heilman KM. Thalamic neglect. Possible role of the medial thalamus and nucleus reticularis in behavior. Arch Neurol 1981; 38: 501-6.

38. Cooke PM, Snider RS. Some cerebellar influences on electrically-induced cerebral seizures. Epilepsia 1955; 4: 19-28.

39. Dow RS, Fernandez-Guardiola A, Manni E. The influence of the cerebellum on experimental epilepsy. Electroencephalogr Clin Neurophysiol 1962; 14: 383-98.

40. Bikson M, Lian J, Hahn PJ, et al. Suppression of epileptiform activity by high frequency sinusoidal fields in rat hippocampal slices. J Physiol 2001; 531: 181-91.

41. Kwan P, Arzimanoglou A, Berg AT, et al. Definition of drug resistant epilepsy: Consensus proposal by the ad hoc Task Force of the ILAE Commission on Therapeutic Strategies. Epilepsia 2010; 51: 1069-77.

42. Berg AT, Vickrey BG, Testa FM, et al. How long does it take for epilepsy to become intractable? A prospective investigation. Ann Neurol 2006; 60: 73-9.

43. Fisher R, Salanova V, Witt T, et al. SANTE Group. Electrical stimulation of the anterior nucleus of thalamus for 
treatment of refractory epilepsy. Epilepsia 2010; 51: 899-908.

44. Boon P, Vonck K, De Herdt V, et al. Deep brain stimulation in patients with refractory temporal lobe epilepsy. Epilepsia 2007; 48: 1551-60.

45. Hodaie M, Wennberg, RA, Dostrovsky JO. Chronic anterior thalamus stimulation for intractable epilepsy. Epilepsia 2002; 43: 603-8.

46. Lim SN, Lee ST, Tsai YT, et al. Electrical stimulation of the anterior nucleus of the thalamus for intractable epilepsy: a long-term follow-up study. Epilepsia 2007; 48: 342-7.

47. Franzini A, Messina G, Marras C, et al. Deep brain stimulation of two unconventional targets in refractory non-resectable epilepsy. Stereotact Funct Neurosurg 2008; 86: 373-81.

48. Benabid AL, Minotti L, Koudsié A, et al. Antiepileptic effect of high-frequency stimulation of the subthalamic nucleus (corpus luysi) in a case of medically intractable epilepsy caused by focal dysplasia: a 30-month follow-up: technical case report. Neurosurgery 2002; 50: 1385-91.

49. Velasco AL, Velasco F, Velasco M, et al. Electrical stimulation of the hippocampal epileptic foci for seizure control: a double-blind, long-term follow-up study. Epilepsia 2007; 48: 1895-903.

50. Wright G, McLellan D, Brice J. A double-blind trial of chronic cerebellar stimulation in twelve patients with severe epilepsy. J Neurol Neurosurg Psychiatry 1984; 47: 769-74.

51. Velasco AL, Velasco M, Velasco F, et al. Subacute and chronic electrical stimulation of the hippocampus on intractable temporal lobe seizures: preliminary report. Arch Med Res 2000; 31: 316-28.

52. Lyons KE, Wilkinson SB, Overman J. Surgical and hardware complications of subthalamic stimulation: a series of 160 procedures. Neurology 2004; 63: 612-6.

53. Bancaud J, Talairach J, Morel P. Ammon's horn and amygdaline nucleus: clinical and electric effects of their stimulation in man. Rev Neurol 1966; 115: 329-52.

54. Boon P, Vonck K, De Herdt V. Deep brain stimulation in patients with refractory temporal lobe epilepsy. Epilepsia 2007; 48: 1551-60.

55. Kenney C, Simpson R, Hunter C, et al. Short-term and long-term safety of deep brain stimulation in the treatment of movement disorders. J Neurosug 2007; 106: 621-5.

56. Kumar K, Toth C, Nath R. Deep brain stimulation for intractable pain: a 15-year experience. Neurosurgery 1997; 40: $736-46$.

57. Dan NG, Wade MJ. The incidence of epilepsy after ventricular shunting procedures. J Neurosurg 1986; 65: 19-21.

58. Coley E, Farhadi R, Lewis S, Whittle IR. The incidence of seizures following deep brain stimulating electrode implantation for movement disorders, pain and psychiatric conditions. Br J Neurosurg 2009; 23: 179-83.

59. Tronnier VM, Staubert A, Hahnel S, Sarem-Aslani A. Magnetic resonance imaging with implanted neurostimulators: an in vitro and in vivo study. Neurosurgery 1999; 44: 118-26.

60. Jankovic J, Cardoso F, Grossman RG, Hamilton WJ. Outcome after stereotactic thalamotomy for parkinsonian, essential and other types of tremor. Neurosurgery 1995; 37: 680-7.

61. Videnovic A, Metman LV. Deep brain stimulation for Parkinson's disease: prevalence of adverse events and need for standardized reporting. Mov Disord 2008; 23: 343-9.
62. Weaver FM, Follett K, Stern M, et al. Bilateral deep brain simula- tion versus best medical therapy for patients with advanced Parkinson disease. JAMA 2009; 301: 63-73.

63. Hariz MI. Complications of deep brain stimulation surgery. Mov Disord 2002; 17 (Suppl 3): S162-6.

64. Lyons K, Koller W, Wiklinson S, Pahwa PR. Surgical and device-related events with deep brain stimulation. Neurology 2001; 56: A147.

65. Kerrigan J, Litt FB, Fisher R. Electrical stimulation of the anterior nucleus of the thalamus for the treatment of intractable epilepsy. Epilepsia 2004; 45: 346-54.

66. Osorio I, Overman J, Giftakis J, et al. High frequency thalamic stimulation for inoperable mesial temporal epilepsy. Epilepsia 2007; 48: 1561-71.

67. Andrade DM, Zumsteg D, Hamani C, et al. Long-term follow-up of patients with thalamic deep brain stimulation for epilepsy. Neurology 2006; 66: 1571-3.

68. Lee KJ, Jang KS, Shon YM. Chronic deep brain stimulation subthalamic and anterior thalamic nuclei for controlling refractory partial epilepsy. Acta Neurochir Suppl (Wien) 2006; 99: 87-91.

69. Boex C, Seeck M, Vulliemoz S, et al. Chronic deep brain stimulation in mesial temporal lobe epilepsy. Seizure 2011; 20: 485-90.

70. Chabardès S, Kahane P, Minotti L, et al. Deep brain stimulation in epilepsy with particular reference to the subthalamic nucleus. Epileptic Disord 2002; Suppl 3: S83-93.

71. Handforth A, DeSalles AA, Krahl SE. Deep brain stimulation of the subthalamic nucleus as adjunct treatment for refractory epilepsy. Epilepsia 2006; 47: 1239-41.

72. Shon YM, Lee KJ, Kim HJ, et al. Effect of chronic deep brain stimulation of the subthalamic nucleus for frontal lobe epilepsy: subtraction SPECT analysis. Stereotact Funct Neurosurg 2005; 83: 84-90.

73. Wille C, Steinhoff BJ, Altenmuller DM, et al. Chronic highfrequency deep-brain stimulation in progressive myoclonic epilepsy in adulthood: report of five cases. Epilepsia 2011; 52: 489-96.

74. Vonck K, Boon P, Achten E. et al. Long-term amygdalohippocampal stimulation for refractory temporal lobe epilepsy. J Ann Neurol 2002; 52: 556-65.

75. Tellez-Zenteno JF, McLachlan RS, Parrent A, et al. Hippocampal electrical stimulation in mesial temporal lobe epilepsy. Neurology 2006; 66: 1490-4.

76. Boon P, Vonck K, De Herdt V, et al. Deep brain stimulation in patients with refractory temporal lobe epilepsy. Epilepsia 2007; 48: 1551-60.

77. McLachlan RS, Pigott S, Tellez-Zenteno JF, Wiebe S, Parrent A. Bilateral hippocampal stimulation for intractable temporal lobe epilepsy: impact on seizures and memory. Epilepsia 2010; 51: 304-7.

78. Miatton M, Van Roost D, Thiery E, et al. The cognitive effects of amygdalohippocampal deep brain stimulation in patients with temporal lobe epilepsy. Epilepsy Behav 2011 Oct 24

79. Velasco R, Velasco M, Ogarrio C, et al. Electrical stimulation of the centromedian thalamic nucleus in the treatment of convulsive seizures: a preliminary report. Epilepsia 1987; 28: 421-30.

80. Velasco $F$, Velasco $M$, Jiménez $F$, et al. Predictors in the treatment of difficult-to-control seizures by electrical stimulation of the centromedian thalamic nucleus. Neurosurgery 2000; 47: 295-304. 
81. Cooper IS, Amin I, Gilman S. The effect of chronic cerebellar stimulation upon epilepsy in man. Trans Am Neurol Assoc 1973; 98: 192-6.

82. Fisher R, Uematsu S, Krauss GL, et al. Placebo-controlled pilot study of centromedian thalamic stimulation in treatment of intractable seizures. Epilepsia 1992; 33: 841-51.

83. Cukiert A, Burattini JA, Cukiert CM, et al. Centro-median stimulation yields additional seizure frequency and attention improvement in patients previously submitted to callosotomy. Seizure 2009; 18: 588-92.

84. Valentin A, Nguyen HQ, Skupenova AM, et al. Centromedian thalamic nuclei deep brain stimulation in refractory status epilepticus. Brain Stimul 2011 Oct 30.

85. Vesper J, Steinhoff B, Rona S, et al. Chronic high-frequency deep brain stimulation of the STN/SNr for progressive myoclonic epilepsy. Epilepsia 2007; 48: 1984-9.

86. Velasco F, Carrillo-Ruiz J, Brito F, et al. Double-blind, randomized controlled pilot study of bilateral cerebellar stimulation for treatment of intractable motor seizures. Epilepsia 2005; 46: 1071-81.

87. Chkhenkeli SA, Chkhenkeli IS. Effects of therapeutic stimulation of nucleus caudatus on epileptic electrical activity of brain in patients with intractable epilepsy. Stereotact Funct Neurosurg 1997; 69: 221-4.

88. Roger $M$, Cadusseau J. Afferents to the zona incerta in the rat: a combined retrograde and anterograde study. J Comp Neurol 1985; 241: 480-92.

89. Heise CE, Mitrofanis J. Evidence for a glutamatergic projection from the zona incerta to the basal ganglia of rats. J Comp Neurol 2004; 468: 482-95.

90. Bartho P, Freund TF, Acsady L. Selective GABAergic innervation of thalamic nuclei from zona incerta. Eur J Neurosci 2002; 16: 999-1014.

91. Sun FT, Morrell MJ, Wharen RE Jr. Responsive cortical stimulation for the treatment of epilepsy. Neurotherapeutics 2008; 5: 68-74.

92. Cukiert A, Cukiert CM, Argentoni-Baldochi M, et al. Intraoperative neurophysiological responses in epileptic patients submitted to hippocampal and thalamic deep brain stimulation. Seizure 2011; 20: 748-53.

93. Boëx C, Vulliémoz S, Spinelli L, Pollo C, Seeck M. High and low frequency electrical stimulation in non-lesional temporal lobe epilepsy. Seizure 2007; 16: 664-9.

94. Tyrand R, Seeck M, Spinelli L, et al. Effects of amygdalahippocampal stimulation on interictal epileptic discharges. Epilepsy Res 2011 doi:10.1016/j.eplepsyres. 2011.10.026.

95. Benabid AL, Pollak P, Gross C, et al. Acute and long-term effects of subthalamic nucleus stimulation in Parkinson's disease. Stereotact Funct Neurosurg 1994; 62: 76-84.

96. Bell E, Mathieu G, Racine E. Preparing the ethical future of deep brain stimulation. Surg Neurol 2009; 72: 577-86.

97. Bell E, Maxwell B, McAndrews MP, Sadikot A, Racine E. Hope and patients' expectations in deep brain stimulation: healthcare providers' perspectives and approaches. J Clin Ethics 2010; 21: 112-24.

98. Rabins P, Appleby BS, Brandt J, et al. Scientific and ethical issues related to deep brain stimulation for disorders of mood, behavior and thought. Arch Gen Psychiatry 2009; 66: 931-7. 